\title{
Coordinating cohesion, co-orientation, and congression during meiosis: lessons from holocentric chromosomes
}

\author{
Mara Schvarzstein, Sarah M. Wignall, and Anne M. Villeneuve ${ }^{1}$ \\ Department of Developmental Biology, Stanford University School of Medicine, Stanford, California 94305, USA; and \\ Department of Genetics, Stanford University School of Medicine, Stanford, California 94305, USA
}

Organisms that reproduce sexually must reduce their chromosome number by half during meiosis to generate haploid gametes. To achieve this reduction in ploidy, organisms must devise strategies to couple sister chromatids so that they stay together during the first meiotic division (when homologous chromosomes separate) and then segregate away from one another during the second division. Here we review recent findings that shed light on how Caenorhabditis elegans, an organism with holocentric chromosomes, deals with these challenges of meiosis by differentiating distinct chromosomal subdomains and remodeling chromosome structure during prophase. Furthermore, we discuss how features of chromosome organization established during prophase affect later chromosome behavior during the meiotic divisions. Finally, we illustrate how analysis of holocentric meiosis can inform our thinking about mechanisms that operate on monocentric chromosomes.

During sexual reproduction, diploid chromosome number must be reduced in half to generate haploid gametes. This reduction in chromosome number is accomplished during meiosis, a specialized cell division program in which a single round of DNA replication is followed by two rounds of chromosome segregation. During prophase of meiosis I, homologous chromosomes pair, align, and undergo crossover recombination between their DNA molecules. These crossovers collaborate with sister chromatid cohesion (SCC) to create temporary physical links, called chiasmata, that connect homologs and allow them to orient and then segregate toward opposite poles of the meiosis I spindle, thereby achieving reduction in ploidy. The reductional meiosis I division is followed by an equational meiosis II division, akin to mitosis, in which sister chromatids are segregated to opposite spindle poles.

Chromosome segregation during meiosis presents two special challenges for the cell division machinery: (1) SCC must be released in two steps. This is necessary to allow

[Keywords: Cohesion; congression; co-oriention; meiosis] ${ }^{1}$ Corresponding author.

E-MAIL villen@cmgm.stanford.edu; FAX (650) 725-7739.

Article is online at http://www.genesdev.org/cgi/doi/10.1101/gad.1863610. release of chiasmata and reductional segregation in meiosis I, while retaining a local region of sister cohesion necessary to align chromosomes and to maintain sister connections until anaphase of meiosis II. (2) Sister chromatids must be temporarily "co-oriented" during meiosis I, so that they move together to the same spindle pole. Sister chromatids must then switch their behavior, becoming "bioriented" on the meiosis II spindle so that they can move apart toward opposite spindle poles.

In organisms with monocentric chromosomes, the single localized centromere serves as the focal point for mechanisms that serve to co-orient sister chromatids at meiosis I and for mechanisms that promote local protection of cohesion to allow two-step release of SCC (Sakuno and Watanabe 2009). In contrast, organisms with holocentric chromosomes, which do not have a localized centromere, cannot rely on a single predefined site to regulate sister chromatid co-orientation and the two-step loss of cohesion during meiosis.

Although monocentric chromosome organization is more familiar to most biologists, holocentric chromosome organization is found in a wide variety of organisms distributed throughout the eukaryotic phylogeny, occurring in plants and in various types of animals including nematodes, arachnids, and several orders of insects (White 1973). Furthermore, monocentric chromosomes themselves differ widely; for example, the sizes of the chromosomal domains that define the centromere in different organisms range over four orders of magnitude, suggesting that it may be misleading to consider monocentric chromosomes as a single class (Ekwall 2004). Moreover, recent work has clearly established that the molecular components of kinetochores are conserved among monocentric and holocentric organisms (Oegema and Hyman 2006), emphasizing the fact that studies of holocentric chromosomes can be illuminating for understanding both diverged and conserved features of chromosome organization and function.

Here we review and synthesize findings from recent work on meiosis in the nematode Caenorhabditis elegans that have shed light on how organisms with holocentric chromosomes deal with the two special challenges of meiosis described above. In particular, we illustrate how 
unique organizational features of C. elegans chromosomes can serve to address these challenges, allowing chromosomes to align and segregate faithfully during the meiotic divisions. Furthermore, we show how conserved chromosomal components can be used in different ways in monocentric versus holocentric chromosomes to achieve the same ends, and we discuss evidence that mechanisms discovered through analysis of holocentric meiosis may also operate on monocentric chromosomes.

\section{Meiotic prophase events prepare chromosomes for the meiotic divisions}

The primary function of meiosis is to reduce ploidy by segregating homologous chromosomes. To achieve this end, homologous chromosomes must first become conjoined in a configuration that enables their subsequent disjunction. In most organisms, this is accomplished during an extended meiotic prophase, through a series of events that results in the creation of a temporary link between the homologs (Fig. 1A).

Following meiotic DNA replication, chromosomes reorganize extensively in preparation for the meiotic divisions (Page and Hawley 2003). The chromosomes begin to condense and develop a specialized axial structure along the lengths of their conjoined sister chromatids; these axial elements are comprised of cohesin complexes and a variety of meiosis-specific structural proteins. Homologous chromosome pairs align along their lengths, and this alignment is stabilized through synapsis; i.e.,

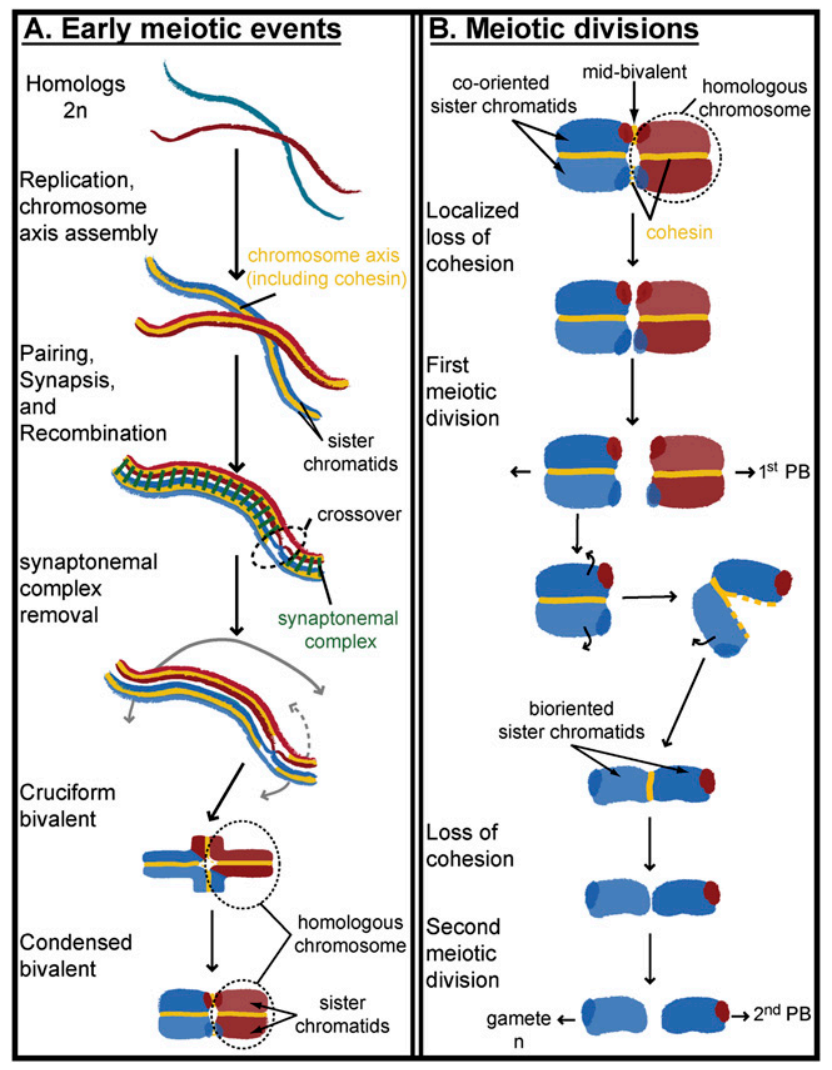

assembly of a proteinaceous structure known as the synaptonemal complex (SC) that links the axes of the aligned homologs. Within this context, crossover recombination occurs between the DNA molecules of nonsister chromatids. Later in prophase, the SC disassembles and chromosomes continue to condense and reorganize into a more compact structure that we will refer to as a bivalent. At this stage, the homologous regions of nonsister chromatids are largely separated from each other, but remain closely juxtaposed at only a few (often one) discrete points known as chiasmata (see the "cruciform bivalent" in Fig. 1A, which has one chiasma). Each chiasma corresponds to a position where a crossover between the homologs, together with SCC flanking the

Figure 1. Key steps in C. elegans oocyte meiosis. This figure diagrams the progression of one pair of homologous chromosomes (depicted in red and blue) through meiosis. (A) Early meiotic events. Following DNA replication, a specialized meiotic chromosome axis structure (shown in yellow) is assembled along the lengths of the sister chromatid pairs. Components of these axial elements include cohesin complexes containing meiosis-specific subunits and members of the meiosis-specific HORMA domain protein family (the four C. elegans paralogs are known as HIM-3, HTP-1, HTP-2, and HTP-3). Homologous chromosomes align along their lengths, and the SC assembles between them to stabilize this alignment. Within this context, crossover recombination events are completed between the DNA molecules of the homologs; the diagram depicts an example in which a single crossover has occurred. This crossover, together with SCC in the chromosomal domains flanking the crossover site, results in a structure known as a chiasma, which maintains the homologs together as a bivalent after the SC is disassembled. As the SC disassembles, the homolog pair undergoes a major structural remodeling and becomes further compacted to yield a bivalent with a cruciform shape. Until this point, the events and chromosome organizational features depicted are typical of meiosis in many organisms, including those with monocentric chromosomes. During C. elegans meiosis, robust crossover regulation mechanisms result in a single crossover between each pair of homologs. Because the crossover site is usually in a substantially off-center position, the resulting cruciform bivalent is asymmetric, containing a short axis and a long axis. Continued condensation during late prophase results in a compact "stir-bar"-shaped bivalent in which the homologous chromosomes are pointing in opposite directions from each other and sister chromatids are co-oriented. By prometaphase, the short arms of the bivalent are so highly compacted that they are not readily discerned; this region of the bivalent is now referred to as the midbivalent. $(B)$ The meiotic divisions. In the first (reductional) division, the homologous chromosomes separate and move to opposite poles of the meiotic spindle. Separation of the homologs during the first division involves departure of cohesin complexes and loss of cohesion at the midbivalent, while cohesin complexes are retained on the bivalent long arms. In oocyte meiosis, one set of homologs will be discarded into the first polar body (PB) and the other will undergo a second, equational division. During the second division, attached sister chromatids are oriented toward opposite poles, with the domains that retain sister cohesion localized at the metaphase plate. In the oocyte, one set of sister chromatids will be discarded into the second polar body, and the other will end up in the gamete nucleus. 
crossover site, maintains association of the homologs as a bivalent until anaphase of meiosis I (Jones 1987).

\section{Crossover-triggered differentiation of chromosome subdomains as a mechanism to direct two-step loss of SCC}

The diagram of the cruciform bivalent in Figure 1A emphasizes the fact that SCC established early in the meiotic program underlies both the connections between sister chromatids and those between homologous chromosomes. Thus the ability to regulate release of cohesion so that it occurs in two steps is key to achieving two successful rounds of chromosome segregation. SCC must be released over a portion of each bivalent to allow dissolution of chiasmata and homolog segregation in meiosis I. At the same time, SCC must be protected over the portion of each homolog that maintains the connections between sister chromatids necessary to promote their alignment and ultimate segregation on the meiosis II spindle. This is accomplished in all studied organisms by locally inhibiting cohesin removal from a subdomain of each chromosome.

In organisms with monocentric chromosomes, localized cohesion protection during meiosis is accomplished by members of the MEI-S332/Shugoshin protein family (Kitajima et al. 2004; Resnick et al. 2006; Sakuno and Watanabe 2009). MEI-S332/Shugoshin proteins are targeted to centromeric and/or pericentromeric domains of chromosomes, where they inhibit degradation of Rec8, a meiosis-specific cohesin complex subunit, at the metaphase/anaphase transition of meiosis I. MEIS332/Shugoshin acts by recruiting protein phosphatase PP2A, which locally maintains Rec8 in a dephosphorylated state, thereby protecting it from cleavage by the separase protease.

In organisms with holocentric chromosomes, the absence of a predefined site for cohesion protection necessitates alternative mechanisms to regulate cohesin loss during meiosis. During C. elegans meiosis, the crossover serves as a key element in this process, linking mechanisms that create chiasmata with mechanisms that determine where cohesion will be released and retained at the meiosis I division. Each of the six C. elegans chromosome pairs usually undergoes a single crossover. Furthermore, since this crossover usually occurs in a substantially off-center position along the length of the chromosome (Barnes et al. 1995), late prophase remodeling results in six cruciform bivalents with distinct long and short arms (Fig. 1A; Albertson et al. 1997). Importantly, the location of the crossover dictates which ends of the chromosomes will be oriented toward the spindle poles and where SCC will be lost at meiosis I (Albertson and Thomson 1993; Albertson et al. 1997). As the position of the crossover is variable from one meiosis to the next, the domains of cohesion retention and loss and the orientation of chromosomes on the spindle are thus also variable.

How does the crossover position direct the subsequent behavior of the chromosomes? The short answer is that the crossover triggers differentiation of the meiotic bi- valent into distinct functional domains; each of these domains harbors a distinct complement of proteins that determines its properties and fate during the meiotic divisions (Fig. 2A). Crossover-triggered differentiation first becomes evident during the late pachytene stage of meiotic prophase, even before the chromosomes have desynapsed. The majority of chromosome axis components (including cohesin complexes and the meiosisspecific HORMA domain proteins HTP-3 and HIM-3) retain relatively uniform distributions along the lengths of chromosome axes as the homologs desynapse and are remodeled during late prophase. Therefore, these proteins localize at the interfaces between sister chromatids on both the long and short arms of the bivalents, generating a cross-like appearance (Chan et al. 2004; Nabeshima et al. 2005). In contrast, several other proteins that had also been distributed along the lengths of the synapsed chromosomes at earlier stages begin to relocalize in a crossover-dependent manner beginning in late pachytene, ultimately becoming concentrated on distinct chromosomal domains. Chromosome axis components HTP-1 and HTP-2 (members of the meiosis-specific HORMA domain family, hereafter referred to as HTP-1/2) and LAB-1 (a nematode-specific protein) become depleted from a domain extending from the presumptive crossover site to the nearest chromosome end, and are retained on the domain extending from the crossover site to the opposite chromosome end (Fig. 2A; de Carvalho et al. 2008; Martinez-Perez et al. 2008). Conversely, SC central region proteins SYP-1, SYP-2, and SYP-3, and crossover-promoting RING finger domain protein $\mathrm{ZHP}-3$ adopt a reciprocal localization; i.e., they are retained on the domain where HTP-1/2 and LAB-1 are depleted (Nabeshima et al. 2005; Smolikov et al. 2007; Bhalla et al. 2008; de Carvalho et al. 2008; Martinez-Perez et al. 2008). The ZHP-3 domain continues to shrink until it becomes concentrated at a single focus, presumably marking the crossover site (Bhalla et al. 2008). These localization patterns persist as chromosomes desynapse and reorganize into the cruciform bivalent structure. Thus, in this configuration, SYP proteins associate with the short arms, where cohesion will be lost at meiosis I (Pasierbek et al. 2001), and HTP-1/2 and LAB-1 associate with the long arms, where cohesion will be retained until meiosis II.

The underlying mechanism by which the meiotic machinery distinguishes which chromosome end is nearest to the crossover, and differentially alters the protein composition of chromosome axis domains in response, remains a mystery. However, we note that there are intriguing parallels between this axis differentiation process and the long-recognized but enigmatic phenomenon of crossover interference (Hillers 2004), which also involves distance-dependent changes in chromosomal properties that are triggered by crossovers.

Despite unanswered questions regarding how reciprocal differentiation of bivalent subdomains arises, we are now beginning to understand how this organization regulates the timing of cohesion loss. As bivalents continue to condense in preparation for the meiotic divisions, the short arms become even more highly compacted, making 


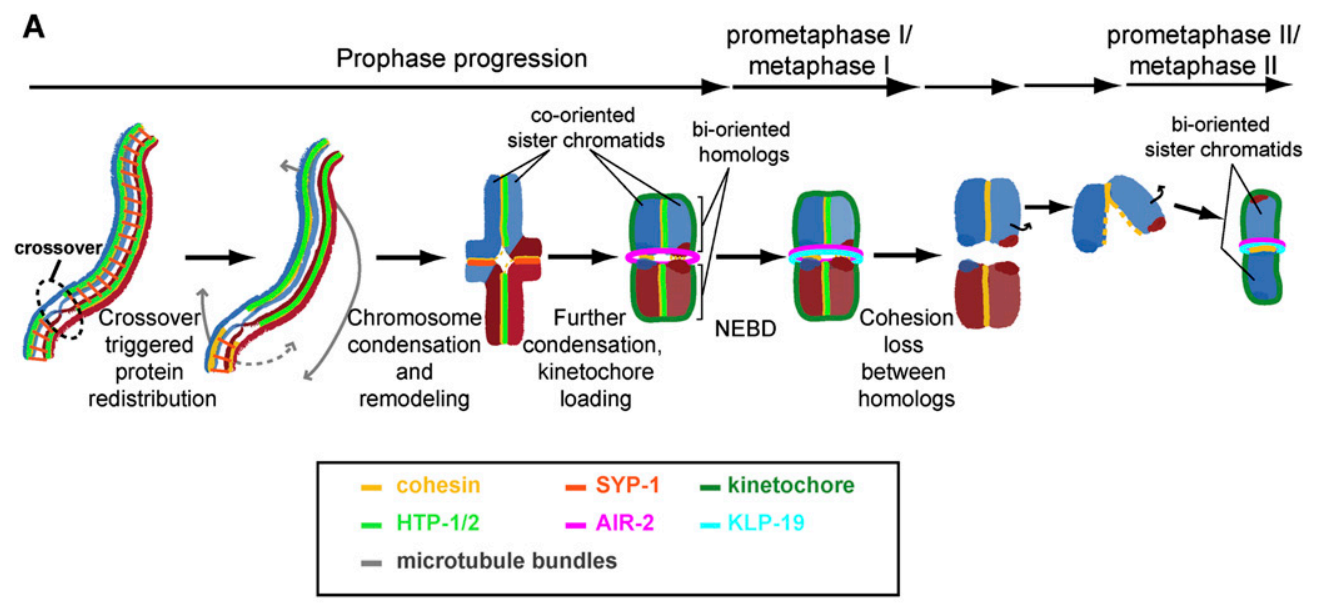

B

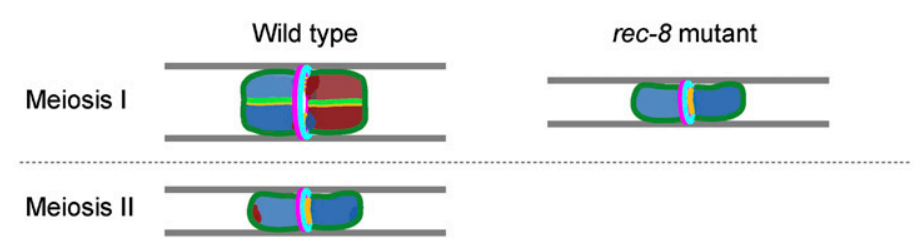

Figure 2. Crossover-triggered differentiation of bivalent subdomains dictates chromosome organization and behavior during the meiotic divisions. (A) Diagram of key steps during C. elegans bivalent differentiation. During most of the pachytene stage of meiotic prophase, SC central region proteins (e.g., SYP-1; refer to the legend for color assignments) and chromosome axis components (e.g., HTP-1/2) are localized along the full length of each aligned and synapsed chromosome pair. Beginning in late pachytene, SC central region proteins and a subset of chromosome axis components (including HTP-1/2) become relocalized in a crossover-dependent manner to reciprocal chromosomal domains: SYP proteins become concentrated in the domain between the crossover and the nearest chromosome end, while HTP-1/2 become concentrated in the domain between the crossover and the opposite end of the chromosome. Upon condensation and reorganization of the homolog pair into the cruciform bivalent configuration, SYP-1 is concentrated on the short arms of the bivalent, while HTP-1/2 are concentrated on the bivalent long arms. SYP-1 departs from the chromosomes as they condense further into the compact bivalent configuration, and another set of proteins (including the Aurora B kinase, AIR-2) becomes concentrated in a ring-like organization around the midbivalent. Concurrently, kinetochore proteins are loaded onto the chromosomes, with outer kinetochore proteins organized in a cup-like structure encasing each homolog. In late prophase, the chromokinesin KLP-19 is diffusely localized throughout the bivalent (not depicted); upon nuclear envelope breakdown (NEBD), AIR-2 directs restriction of KLP-19 to a ring around the midbivalent. Cohesin complexes and SCC in the midbivalent region are lost at anaphase I onset to allow homolog segregation, and HTP-1/2 are lost from the reciprocal chromosomal domains in anticipation of meiosis II. AIR-2 and KLP-19 also depart from the chromosomes during anaphase I. During meiosis II, AIR-2 and KLP-19 become relocalized in rings surrounding the sister chromatid interface, where cohesin is now concentrated, and sister chromatids are encased separately by kinetochore components. (B) Ensheathment of chromosomes by lateral microtubule bundles. Diagrams depict the predominant mode of chromosome/microtubule association during prometaphase and metaphase of C. elegans oocyte meiosis. Bivalents (in meiosis I) and attached sister chromatid pairs (in meiosis II) are ensheathed by bundles of microtubules that run laterally along their sides. Chromokinesin KLP-19 localized in a ring is positioned to mediate movement of chromosomes along these lateral microtubule bundles. The organization of the univalent chromosome at meiosis I in the rec-8 mutant resembles that of a normal meiosis II chromosome.

them difficult to distinguish (Fig. 1). During this time, SYP proteins depart and are replaced by a new cohort of proteins-including Aurora B kinase AIR-2 and other components of the chromosome passenger complex (CPC) - that become localized at the compacted short arm domain, now referred to as the midbivalent (Fig. 2A; Kaitna et al. 2002; Rogers et al. 2002; Nabeshima et al. 2005). In C. elegans, AIR-2 is required for cohesion loss and chromosome segregation at meiosis I (Kaitna et al. 2002; Rogers et al. 2002), and it is thought to promote cohesin removal through direct phosphorylation of REC-8 (Rogers et al. 2002). Conversely, several lines of evidence implicate HTP-1/2 and LAB-1 in protecting cohesion on the long arms of the bivalent (de Carvalho et al. 2008; Martinez-
Perez et al. 2008). HTP-1/2 and LAB-1 are ideally positioned for this role, colocalizing with REC- 8 on the long arms of the bivalent. Importantly, both HTP-1 and LAB-1 inhibit localization of AIR-2 in this domain. Furthermore, the kinetics of departure of HTP-1/2 and LAB-1 meet expectations for proteins that function in localized cohesion protection during meiosis I: They persist strongly on chromosomes through the metaphase-to-anaphase transition of meiosis I, then disappear at anaphase I to allow loss of the remaining cohesion at anaphase II (de Carvalho et al. 2008; Martinez-Perez et al. 2008). Finally, depletion of HTP-1/2 results in both premature loss of REC-8 and premature separation of sister chromatids at anaphase of meiosis I, indicating a role for HTP-1/2 in 
inhibiting cohesion release (Martinez-Perez et al. 2008). Thus, crossover-triggered bivalent differentiation regulates cohesion release by concentrating proteins that either promote or inhibit cohesion release to the appropriate chromosomal domains.

The proposed roles of HTP- $1 / 2$ and LAB- 1 in conferring localized protection of SCC until meiosis II are analogous to those of MEI-S332/Shugoshin in protecting centromeric SCC in organisms with monocentric chromosomes. However, the sole C. elegans Shugoshin homolog SGO-1 is not required for cohesion protection during meiosis, indicating either that it does not play such a role or that its contribution is masked by the predominance of the HTP1/2/LAB-1-based mechanism (de Carvalho et al. 2008; Severson et al. 2009). Conversely, identification of a role for HTP-1/2 in localized cohesion protection in C. elegans raises the question of whether other members of the meiosis-specific HORMA domain protein family might participate in cohesion protection in other organisms. In this regard, it is interesting to note that meiosis-specific HORMA proteins PAIR-2 in rice (Nonomura et al. 2006) and HORMAD1 and HORMAD2 in mice (Wojtasz et al. 2009) also localize at the centromeres of metaphase I meiotic bivalents. However, the functional significance of this localization is not yet known.

In light of the fact that different mechanisms for localized SCC protection appear to predominate for holocentric versus monocentric chromosomes, it is noteworthy that Aurora B kinases participate in regulating two-step loss of SCC in both instances. However, whereas C. elegans AIR-2 promotes cohesion release at meiosis I, Drosophila and yeast Aurora B homologs protect cohesion locally, by maintaining localization of MEIS332/Shugoshin and/or the PP2A regulatory subunit at centromeres (Resnick et al. 2006; Yu and Koshland 2007). As Aurora B kinases have been implicated in orchestrating many additional aspects of cell division (Ruchaud et al. 2007), it is possible that this reversal of the role for AIR-2 might be an evolutionary consequence of constraints on AIR-2 localization imposed by other requirements of holocentric chromosome organization (e.g., see the "Biorienting Bivalents and Congressing Chromosomes" section below). Furthermore, this role reversal might also account for the predominance of one strategy over another in regulating two-step loss of SCC.

\section{Co-orienting sister chromatids at meiosis I}

While up to this point we focused on mechanisms by which two-step loss of SCC can be accomplished with chromosomes lacking localized centromeres, the second major challenge of the meiotic divisions that must be addressed is the co-orientation of sister chromatids at meiosis I. In organisms with monocentric chromosomes, this challenge is overcome by mechanisms ensuring that the centromeres of sister chromatids behave as a single functional unit with a single functional kinetochore mediating meiosis I spindle attachment (Sakuno and Watanabe 2009). This coordination serves to ensure that pairs of sister chromatids move in unison to the same pole (while homologs segregate to opposite poles) at meiosis I. In considering how sister chromatid co-orientation might be accomplished in the context of holocentric chromosomes, it is crucial to understand how the kinetochore components are organized on the chromosomes. Both electron microscopy (EM) studies and immunofluorescence localization of kinetochore proteins during C. elegans meiosis have revealed outer kinetochore components organized as cup-like structures encapsulating each half-bivalent (at meiosis I) or each chromatid (at meiosis II) (Fig. 2; Howe et al. 2001; Monen et al. 2005). This organization suggests the hypothesis that encasement of sister chromatids together within the same compartment may help to promote their behavior as a single functional unit during the meiosis I division. But what causes the sister chromatids to be encased together in the first place?

A recent study published in Genes \& Development by Severson et al. (2009) identified cohesin complexes containing the meiosis-specific kleisin subunit REC-8 as a key player in sister chromatid co-orientation at meiosis I. Because of a prior defect in meiotic crossing-over, homologous chromosomes are not connected by chiasmata in rec-8 mutant oocytes (Pasierbek et al. 2001; Severson et al. 2009). In many other mutants that lack meiotic crossovers, the unattached homologous chromosomes (known as univalents) appear to be lost or segregate randomly at meiosis I. However, univalents in rec-8 mutant oocytes consistently segregate equationally (i.e., sister chromatids segregate to opposite poles) in meiosis I, just as they would normally do in mitosis or meiosis II (Severson et al. 2009). Cytological examination of chromosomes at late prophase and prometaphase I provided a ready explanation for this behavior: Individual chromosomes in rec-8 mutants enter the meiosis I division with an organization and appearance that resemble that of normal meiosis II chromosomes (Fig. 2A,B). Sister chromatids are largely separated and oriented away from each other, and proteins and chromatin marks normally concentrated at the midbivalent in meiosis I localize to the remaining sister chromatid interface (Severson et al. 2009). As expected based on both this organization and the segregation behavior in rec-8 mutant meiosis, sister chromatids are encased separately by outer kinetochore proteins when they enter meiosis I (Fig. 2B; A Severson and B Meyer, pers. comm.).

A central role for C. elegans REC-8 in promoting sister chromatid co-orientation in meiosis I is especially intriguing in light of the fact that Rec8 has also been implicated recently in promoting sister chromatid co-orientation during meiosis I in both Arabidopsis (Chelysheva et al. 2005; d'Erfurth et al. 2009) and fission yeast (Sakuno et al. 2009). In Arabidopsis, simultaneous loss of both REC8 and SPO11-1 results in equational segregation at meiosis I, paralleling the results seen in C. elegans. Furthermore, fission yeast Rec8 has been shown to act within the core centromere domain itself, collaborating with a nonconserved factor (Moal) to define a kinetochore geometry that favors co-orientation of sister centromeres to the same spindle pole. The fact that these diverse organisms all use 
specialized meiotic cohesin complexes to confer consolidated behavior of sister chromatids at meiosis I suggests that this strategy is an ancient innovation of the cell division machinery linked to the evolution of meiosis from mitosis.

\section{Functional diversification of conserved chromosome axis components}

Severson's analysis of meiosis in mutants lacking REC-8 suggested the existence of additional cohesin complexes operating during meiosis (Severson et al. 2009). Although meiosis I sister chromatid co-orientation was lost in the rec- 8 mutants, sister chromatids nevertheless remained connected through metaphase I, enabling their equational segregation at anaphase I. Furthermore, this phenotype is distinct from the phenotype observed in mutants lacking the SCC-3 cohesin component, in which axial element and SC assembly fail and detached sister chromatids and chromosome fragments are observed in late prophase (Pasierbek et al. 2003; Wang et al. 2003; Goodyer et al. 2008). Indeed, Severson et al. (2009) identified two additional kleisin subunits, the nearly identical COH-3 and $\mathrm{COH}-4$ proteins, that function in combination with REC-8 to mediate SCC in meiosis. Cohesin complexes involving all three different kleisins contribute to axial element formation, as failure of axial element assembly is observed only when all three are eliminated simultaneously. The different meiotic cohesin complexes also have specialized, nonredundant functions; in particular, only REC-8 can function to ensure chromatid co-orientation at meiosis I (Severson et al. 2009).

Following the equational segregation of sister chromatids in rec-8 mutants, oocytes attempt a second meiotic division but fail to extrude a second polar body (Severson et al. 2009). Although the basis of this failure is not yet fully understood, a clear consequence is that the resulting embryos retain a diploid set of maternal chromosomes. Severson et al. (2009) seized on this property and exploited it as the basis of a clever genetic screen to identify other components required for the persistence of SCC until anaphase II. Interestingly, this screen identified HTP-3, a previously known meiotic chromosome axis protein (MacQueen et al. 2005; Goodyer et al. 2008).

HTP-3 is one of four C. elegans members of the aforementioned meiosis-specific HORMA domain family that also includes HTP-1/2. Members of this protein family have been implicated in a myriad of roles in nearly all aspects of meiotic chromosome function, including homolog pairing and SC assembly, initiation of meiotic recombination, inhibition of nonhomologous synapsis, inhibition of recombination between sister chromatids, and localized protection of cohesion (Couteau et al. 2004; Couteau and Zetka 2005; Martinez-Perez and Villeneuve 2005; Goodyer et al. 2008; Martinez-Perez et al. 2008). Like the different meiotic cohesin complexes, the different HORMA paralogs have been functionally diversified and have both partially redundant and specialized roles. The analysis by Severson et al. (2009) has added a new and unexpected role to the list. While it was known that cohesin complexes were required for the localization of HTP-3 (and the other paralogs) to meiotic chromosome axes (Goodyer et al. 2008), Severson et al. (2009) found that cohesins did not localize to cytologically detectable chromosome axes in htp-3-null mutants. This observation indicates that cohesins and HTP-3 are interdependent for localization to and assembly of meiotic chromosome axes. Their roles are not completely equivalent, however, as sister chromatids maintain tighter connections during late prophase/prometaphase in htp-3 mutants than in rec- 8 mutants, and a combination of cytological and genetic experiments indicate that some residual function of both REC-8-containing and $\mathrm{COH}-3$ / 4-containing cohesin complexes persists in htp-3 mutants (Severson et al. 2009).

Interdependence of HTP-3 and cohesin complexes suggests a new view of axial element assembly. Rather than being built on a preformed "foundation" of cohesin, it is more likely that the meiotic chromosome axis coalesces as a discrete linear entity at the onset of meiotic prophase through simultaneous recruitment of HORMA proteins and cohesin complexes, some of which (i.e., HTP-3 and cohesin) are preloaded onto chromosomes prior to meiotic prophase entry.

\section{Biorienting bivalents and congressing chromosomes}

The distinctive organizational features of C. elegans meiotic chromosomes that we discussed thus far raise another key question about the meiotic divisions. Namely, how do such chromosomes become bioriented and achieve metaphase alignment on meiotic spindles?

A recent report from our laboratory revealed one way in which the organization of meiotic bivalents can serve to influence the behavior of these chromosomes on the spindle (Wignall and Villeneuve 2009). In particular, we investigated how bivalents achieve proper biorientation and alignment during $C$. elegans oocyte meiosis, where the spindles lack centrosomes. Earlier studies had shown that bivalents orient axially on these acentrosomal spindles, with their long axes parallel to the spindle microtubules and their midbivalent domains at the metaphase plate (Albertson and Thomson 1993). In the new study, high-resolution imaging of prometaphase and metaphase spindles revealed a surprising organization of microtubules with respect to chromosomes. Specifically, bivalents (in meiosis I) and attached sister chromatid pairs (in meiosis II) are ensheathed by robust microtubule bundles that run along their sides, parallel to their long axes, while microtubule density is low at chromosome ends (Fig. 2B). Since the organizational features of the bivalent discussed earlier result in the two homologs being largely oriented in opposite directions throughout the process of spindle assembly (diagrammed in Fig. 2), ensheathment of bivalents by lateral microtubule bundles will naturally result in the homologs becoming bioriented (i.e., pointing toward opposite poles). Interestingly, we also found that the chromokinesin KLP-19 becomes localized in a ring structure around the midbivalent region, where it is in a position to mediate movement along the lateral microtubule 
bundles (Fig. 2). Depletion of this motor caused chromosome alignment defects on metaphase-arrested spindles, indicating that it plays a role in chromosome congression. Since KLP-19 provides a polar ejection force during both mitosis (Powers et al. 2004) and oocyte meiosis (Wignall and Villeneuve 2009), we propose a model in which KLP19 pushes bivalents toward the metaphase plate, where they align between overlapping microtubule bundles. Importantly, localization of this motor to midbivalent rings appears to be a consequence of chromosome organizational features established during prophase. We found that AIR-2 and other CPC components are similarly arranged in rings at the midbivalent, and the CPC is required for targeting of KLP-19 to midbivalent rings.

The low density of microtubules at bivalent ends suggests that lateral interactions are the predominant mode of chromosome/microtubule association during spindle assembly and chromosome alignment in $C$. elegans oocyte meiosis. Interestingly, this aspect of spindle organization may be a common feature of oocyte meiosis, as EM studies of mouse oocyte spindles in prometaphase did not detect kinetochore-microtubuleend interactions, leading Brunet et al. (1999) to propose that congression occurs without a contribution from kinetochore fibers, and raising the possibility that lateral microtubule bundles may instead facilitate this process as they do in C. elegans. We wish to note, however, that our model is fully compatible with the possibility that kinetochore/microtubule interactions may also play roles in chromosome alignment that collaborate with the KLP19-based mechanism. It is possible that a small number of microtubules might form functional attachments with bivalent ends. Moreover, the organization of holocentric chromosomes (where kinetochore proteins are present on the sides of the bivalent) (Fig. 2) could permit kinetochore components to play a role in mediating lateral microtubule associations.

Some of the features of chromosome/microtubule organization discussed above may be especially valuable when dealing with the problem of segregating chromosomes on acentrosomal spindles, since these structures form in the absence of predefined poles and therefore may adopt a distinct configuration. In support of this idea, imaging of spindles in spermatocyte meiosis (which contain centrosomes) revealed high microtubule density adjacent to the kinetochores at the bivalent ends, suggesting that spermatocytes may differ from oocytes with respect to the predominant mode of chromosome association with spindle microtubules (Wignall and Villeneuve 2009). Intriguingly, Shakes et al. (2009) recently conducted a detailed characterization of meiotic progression in spermatocytes that revealed some features of bivalent organization that may influence how chromosome segregation is achieved in these cells. While bivalents in spermatocytes share many organizational features with oocyte chromosomes (including KLP-19 and AIR-2 concentrated at the midbivalent), this study identified some interesting differences in kinetochore composition and organization. Future studies comparing oocytes and spermatocytes will be crucial for dissecting how differences in bivalent organization influence chromosome behavior on the spindle and promote proper chromosome segregation during meiosis.

\section{Structural features of bivalents that facilitate the second meiotic division}

After anaphase I, sister chromatids must switch from an organization in which they move together as a unit toward the same spindle pole to an organization in which they are bioriented and move to opposite spindle poles. An unexplained feature of this transition during $C$. elegans meiosis was the fact that the chromatid ends located at the outside tips of the bivalent long arms, which lead the homologs toward the spindle poles in the first meiotic division, become localized at the metaphase plate in meiosis II (as diagrammed in Figs. 1B, 2A; Albertson and Thomson 1993). A likely mechanism for this transition in chromosome organization was provided recently by high-resolution imaging of late prophase I and prometaphase I bivalents (de Carvalho et al. 2008; Martinez-Perez et al. 2008).

Published images of late prophase I bivalents give the impression that chromosome axis proteins at this stage are organized in linear structures along the sister chromatid interfaces (as diagrammed in Figs. 1B, 2A). However, rotation of high-resolution images in which bivalents show linear localization of axis proteins reveals that these proteins are actually present in a loop-like organization on the bivalent long arms (Fig. 3; de Carvalho et al.
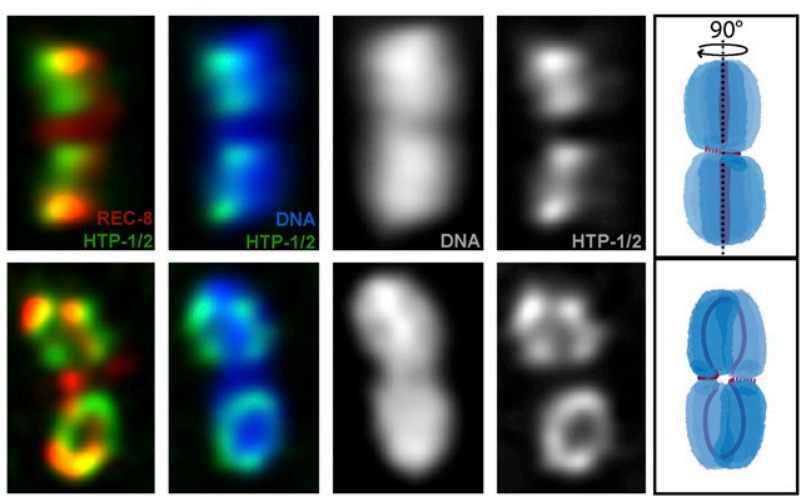

Figure 3. Localization of lateral element proteins in a loop-like organization in the late prophase I bivalent. Immunofluorescence images of a late prophase bivalent and diagrammatic depictions of a model of the bivalent structure. The bivalent depicted in the top panels has been rotated $90^{\circ}$ about its long axis to yield the images and diagrams in the bottom panels. In the top panels, REC- 8 can be detected on both the short and long axes of the bivalent in an apparent cruciform structure, and HTP-1/2 appear as a linear structure along the long arms of the bivalent. (Bottom panels) Upon rotation of the bivalent, the loop-like localization of REC- 8 and HTP-1/2 on the long arms of the bivalent becomes apparent. In the bottom diagram, we depict our interpretation of these images: REC-8 is localized on the long arms to a separate axial structure for each of the two sister chromatids, and these REC- 8 domains are joined only at the tips of the bivalent. 
2008; Martinez-Perez et al. 2008). This organization is seen by both immunofluorescence and live imaging of GFP-tagged axis proteins (M Schvarzstein, unpubl.). Sister chromatids are already largely resolved by this stage, as revealed by DNA staining and immunofluorescence of condensin II components (Chan et al. 2004; Nabeshima et al. 2004, 2005). Thus, we interpret the loop-like organization of REC-8, HTP-1/2, and LAB-1 to represent localization of these proteins to two largely separated axial structures (each associated with one sister chromatid) that are closely associated only near the bivalent ends (see diagram in Fig. 3). This interpretation implies that most of the cohesin protein on the long arms of the bivalent may not confer cohesion between sister chromatids at this stage. Instead, we suggest that cohesion in the late prophase I bivalent is concentrated predominantly at the midbivalent (where it will be released at the onset of anaphase I) and near the ends of the long arms located at the outside tips of the bivalent (where sister chromatids will remain connected until anaphase II) (see diagram in Fig. 3). According to our view, restriction of SCC to a small domain near the end of the bivalent long arm would prepare the chromosomes for both reorienting of sister chromatids and rapid separation of sisters in anaphase II (as the sisters are already separated along most of their lengths). The reorganization of sister chromatids into the metaphase II configuration must take place rapidly, as the kinetochores transiently disassemble between the first and second meiotic divisions (McNally and McNally 2005; Monen et al. 2005). During this brief period $(<8 \mathrm{~min}$ between anaphase I and metaphase II), the proposed organization of sister chromatids would enable them to rapidly become oriented away from each other while remaining associated only at a small domain, ready to segregate to opposite spindle poles (Fig. 2A).

Interestingly, during both sperm and oocyte meiosis in mice, cohesion components become partially lost from most of the chromosomes but remain concentrated at the centromeres prior to metaphase I (Kudo et al. 2006; Viera et al. 2007). Thus, it appears that substantial resolution of SCC during late prophase/prometaphase I, in preparation for the second meiotic division, is not limited to organisms with holocentric chromosomes and is likely to be a widespread feature of the meiotic program.

\section{Concluding remarks}

We illustrated here the strategies used by C. elegans to address the special challenges of chromosome segregation during meiosis in the context of holocentric chromosome organization. In lieu of a predefined site to serve as a control center for regulating events during the meiotic divisions, C. elegans instead uses one of the most fundamental features of the meiotic program, the crossover, to trigger differentiation of the bivalent into distinct chromosomal subdomains. This differentiation process includes changes in the molecular composition, structural organization, and subsequent functional behavior of the different chromosomal domains. In effect, the single crossover on each chromosome pair fulfills the need for a single organizing center that will dictate unambiguously how chromosomes will align, orient, and segregate on the meiosis I and meiosis II spindles. Importantly, much of the functional differentiation of distinct chromosomal subdomains is accomplished using evolutionarily conserved proteins, including meiosis-specific HORMA domain proteins, REC-8-containing cohesin complexes, and the Aurora B kinase, sometimes deployed in seemingly unconventional ways. This suggests that many components of the meiotic machinery can be viewed as elements of a modular toolkit that can be adapted to function in a variety of different physiological contexts. However, it also raises the possibility that multiple mechanisms may operate in parallel to ensure the fidelity of key meiotic events, with different mechanisms predominating in different organisms. Finally, many of the key players involved in conferring specialized properties to distinct domains of meiotic chromosomes in C. elegans (particularly the cohesin complexes and HORMA proteins) have themselves been functionally diversified through gene duplication and sequence divergence. Such functional diversification of conserved protein complexes is emerging as a widespread theme in the meiosis field, particularly in plants and animals, which commonly have multiple HORMA paralogs and multiple distinct cohesin complexes. The C. elegans work exemplifies how functional diversification of conserved chromosomal proteins can provide a means to repeatedly modify the organization of chromosomes and the relationships between sister chromatids. This diversification can serve both to confer different properties at different times during the meiotic program and to enable distinct behaviors for different chromosomal domains.

\section{Acknowledgments}

We thank A. Severson and two anonymous reviewers for comments that improved the manuscript. M.S. holds a CIHR Post-doctoral Fellowship, and S.M.W. is a Special Fellow of the Leukemia and Lymphoma Society. Our research is supported by NIH R01 grants GM53804 and GM67268 to A.M.V.

\section{References}

Albertson DG, Thomson JN. 1993. Segregation of holocentric chromosomes at meiosis in the nematode Caenorhabditis elegans. Chromosome Res 1: 15-26.

Albertson D, Rose A, Villeneuve A. 1997. Chromosome organization, mitosis and meiosis. In C. elegans II (eds. DL Riddle et al.), pp. 47-48. Cold Spring Harbor Laboratory Press, Cold Spring Harbor, NY.

Barnes TM, Kohara Y, Coulson A, Hekimi S. 1995. Meiotic recombination, noncoding DNA and genomic organization in Caenorhabditis elegans. Genetics 141: 159-179.

Bhalla N, Wynne DI, Jantsch V, Dernburg AF. 2008. ZHP-3 acts at crossovers to couple meiotic recombination with synaptonemal complex disassembly and bivalent formation in $C$. elegans. PLoS Genet 4: e1000235. doi: 10.1371/journal.pgen. 1000235.

Brunet S, Maria AS, Guillaud P, Dujardin D, Kubiak JZ, Maro B. 1999. Kinetochore fibers are not involved in the formation of the first meiotic spindle in mouse oocytes, but control the exit from the first meiotic M phase. J Cell Biol 146: 1-12. 
Chan RC, Severson AF, Meyer BJ. 2004. Condensin restructures chromosomes in preparation for meiotic divisions. J Cell Biol 167: 613-625.

Chelysheva L, Diallo S, Vezon D, Gendrot G, Vrielynck N, Belcram K, Rocques N, Marquez-Lema A, Bhatt AM, Horlow C, et al. 2005. AtREC8 and AtSCC3 are essential to the monopolar orientation of the kinetochores during meiosis. I Cell Sci 118: 4621-4632.

Couteau F, Zetka M. 2005. HTP-1 coordinates synaptonemal complex assembly with homolog alignment during meiosis in C. elegans. Genes \& Dev 19: 2744-2756.

Couteau F, Nabeshima K, Villeneuve A, Zetka M. 2004. A component of $C$. elegans meiotic chromosome axes at the interface of homolog alignment, synapsis, nuclear reorganization, and recombination. Curr Biol 14: 585-592.

de Carvalho CE, Zaaijer S, Smolikov S, Gu Y, Schumacher JM, Colaiacovo MP. 2008. LAB-1 antagonizes the Aurora B kinase in C. elegans. Genes \& Dev 22: 2869-2885.

d'Erfurth I, Jolivet S, Froger N, Catrice O, Novatchkova M, Mercier R. 2009. Turning meiosis into mitosis. PLoS Biol 7: e1000124. doi: 10.1371/journal.pbio.1000124.

Ekwall K. 2004. The roles of histone modifications and small RNA in centromere function. Chromosome Res 12: 535-542.

Goodyer W, Kaitna S, Couteau F, Ward JD, Boulton SJ, Zetka M. 2008. HTP-3 links DSB formation with homolog pairing and crossing over during C. elegans meiosis. Dev Cell 14: 263-274.

Hillers KJ. 2004. Crossover interference. Curr Biol 14: R1036R1037. doi: 10.1016/j.cub.2004.11.038.

Howe M, McDonald KL, Albertson DG, Meyer BJ. 2001. HIM-10 is required for kinetochore structure and function on Caenorhabditis elegans holocentric chromosomes. J Cell Biol 153: $1227-1238$.

Jones GH. 1987. Chiasmata. In Meiosis (ed. EP Moens), pp. 213244. Academic Press, Orlando, FL.

Kaitna S, Pasierbek P, Jantsch M, Loidl J, Glotzer M. 2002. The aurora B kinase AIR-2 regulates kinetochores during mitosis and is required for separation of homologous Chromosomes during meiosis. Curr Biol 12: 798-812.

Kitajima TS, Kawashima SA, Watanabe Y. 2004. The conserved kinetochore protein shugoshin protects centromeric cohesion during meiosis. Nature 427: 510-517.

Kudo NR, Wassmann K, Anger M, Schuh M, Wirth KG, Xu H, Helmhart W, Kudo H, McKay M, Maro B, et al. 2006. Resolution of chiasmata in oocytes requires separase-mediated proteolysis. Cell 126: 135-146.

MacQueen AJ, Phillips CM, Bhalla N, Weiser P, Villeneuve AM, Dernburg AF. 2005. Chromosome sites play dual roles to establish homologous synapsis during meiosis in C. elegans. Cell 123: 1037-1050.

Martinez-Perez E, Villeneuve AM. 2005. HTP-1-dependent constraints coordinate homolog pairing and synapsis and promote chiasma formation during C. elegans meiosis. Genes \& Dev 19: 2727-2743.

Martinez-Perez E, Schvarzstein M, Barroso C, Lightfoot J, Dernburg AF, Villeneuve AM. 2008. Crossovers trigger a remodeling of meiotic chromosome axis composition that is linked to two-step loss of sister chromatid cohesion. Genes \& Dev 22: 2886-2901.

McNally KL, McNally FJ. 2005. Fertilization initiates the transition from anaphase I to metaphase II during female meiosis in C. elegans. Dev Biol 282: 218-230.

Monen J, Maddox PS, Hyndman F, Oegema K, Desai A. 2005. Differential role of CENP-A in the segregation of holocentric C. elegans chromosomes during meiosis and mitosis. Nat Cell Biol 7: 1248-1255.
Nabeshima K, Villeneuve AM, Hillers KJ. 2004. Chromosomewide regulation of meiotic crossover formation in Caenorhabditis elegans requires properly assembled chromosome axes. Genetics 168: 1275-1292.

Nabeshima K, Villeneuve AM, Colaiacovo MP. 2005. Crossing over is coupled to late meiotic prophase bivalent differentiation through asymmetric disassembly of the SC. J Cell Biol 168: 683-689.

Nonomura K, Nakano M, Eiguchi M, Suzuki T, Kurata N. 2006. PAIR2 is essential for homologous chromosome synapsis in rice meiosis I. J Cell Sci 119: 217-225.

Oegema K, Hyman AA. 2006. Cell division. In WormBook (ed. The C. elegans Research Community), WormBook. doi: 10.1895/wormbook.1.72.1; http://www.wormbook.org.

Page SL, Hawley RS. 2003. Chromosome choreography: The meiotic ballet. Science 301: 785-789.

Pasierbek P, Jantsch M, Melcher M, Schleiffer A, Schweizer D, Loidl J. 2001. A Caenorhabditis elegans cohesion protein with functions in meiotic chromosome pairing and disjunction. Genes \& Dev 15: 1349-1360.

Pasierbek P, Fodermayr M, Jantsch V, Jantsch M, Schweizer D, Loidl J. 2003. The Caenorhabditis elegans SCC-3 homologue is required for meiotic synapsis and for proper chromosome disjunction in mitosis and meiosis. Exp Cell Res 289: 245255.

Powers J, Rose DJ, Saunders A, Dunkelbarger S, Strome S, Saxton WM. 2004. Loss of KLP-19 polar ejection force causes misorientation and missegregation of holocentric chromosomes. J Cell Biol 166: 991-1001.

Resnick TD, Satinover DL, MacIsaac F, Stukenberg PT, Earnshaw WC, Orr-Weaver TL, Carmena M. 2006. INCENP and Aurora B promote meiotic sister chromatid cohesion through localization of the Shugoshin MEI-S332 in Drosophila. Dev Cell 11: $57-68$.

Rogers E, Bishop JD, Waddle JA, Schumacher JM, Lin R. 2002. The aurora kinase AIR-2 functions in the release of chromosome cohesion in Caenorhabditis elegans meiosis. I Cell Biol 157: 219-229.

Ruchaud S, Carmena M, Earnshaw WC. 2007. Chromosomal passengers: Conducting cell division. Nat Rev Mol Cell Biol 8: 798-812.

Sakuno T, Watanabe Y. 2009. Studies of meiosis disclose distinct roles of cohesion in the core centromere and pericentromeric regions. Chromosome Res 17: 239-249.

Sakuno T, Tada K, Watanabe Y. 2009. Kinetochore geometry defined by cohesion within the centromere. Nature 458: 852-858.

Severson AF, Ling L, van Zuylen V, Meyer BJ. 2009. The axial element protein HTP-3 promotes cohesin loading and meiotic axis assembly in C. elegans to implement the meiotic program of chromosome segregation. Genes \& Dev 23: 1763-1778.

Shakes DC, Wu JC, Sadler PL, Laprade K, Moore LL, Noritake A, Chu DS. 2009. Spermatogenesis-specific features of the meiotic program in Caenorhabditis elegans. PLoS Genet 5: e1000611. doi: 10.1371/journal.pgen.1000611.

Smolikov S, Eizinger A, Schild-Prufert K, Hurlburt A, McDonald K, Engebrecht J, Villeneuve AM, Colaiacovo MP. 2007. SYP-3 restricts synaptonemal complex assembly to bridge paired chromosome axes during meiosis in Caenorhabditis elegans. Genetics 176: 2015-2025.

Viera A, Gomez R, Parra MT, Schmiesing JA, Yokomori K, Rufas JS, Suja JA. 2007. Condensin I reveals new insights on mouse meiotic chromosome structure and dynamics. PLoS One 2: e783. doi: 10.1371/journal.pone.0000783.

Wang F, Yoder J, Antoshechkin I, Han M. 2003. Caenorhabditis elegans EVL-14/PDS-5 and SCC-3 are essential for sister 
Schvarzstein et al.

chromatid cohesion in meiosis and mitosis. Mol Cell Biol 23: 7698-7707.

White MJD. 1973. Animal cytology and evolution. Cambridge University Press, London.

Wignall SM, Villeneuve AM. 2009. Lateral microtubule bundles promote chromosome alignment during acentrosomal oocyte meiosis. Nat Cell Biol 11: 839-844.

Wojtasz L, Daniel K, Roig I, Bolcun-Filas E, Xu H, Boonsanay V, Eckmann CR, Cooke HJ, Jasin M, Keeney S, et al. 2009. Mouse HORMAD1 and HORMAD2, two conserved meiotic chromosomal proteins, are depleted from synapsed chromosome axes with the help of TRIP13 AAA-ATPase. PLoS Genet 5: e1000702. doi: 10.1371/journal.pgen.1000702.

Yu HG, Koshland D. 2007. The Aurora kinase Ipll maintains the centromeric localization of PP2A to protect cohesin during meiosis. J Cell Biol 176: 911-918. 


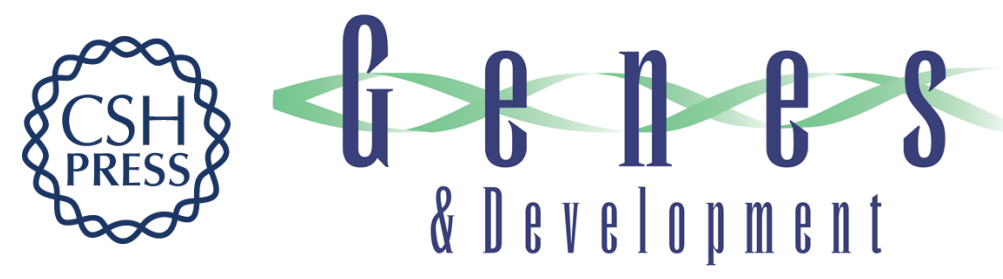

\section{Coordinating cohesion, co-orientation, and congression during meiosis: lessons from holocentric chromosomes}

Mara Schvarzstein, Sarah M. Wignall and Anne M. Villeneuve

Genes Dev. 2010, 24:

Access the most recent version at doi:10.1101/gad.1863610

References This article cites 42 articles, 17 of which can be accessed free at: http://genesdev.cshlp.org/content/24/3/219.full.htmI\#ref-list-1

License

Email Alerting

Receive free email alerts when new articles cite this article - sign up in the box at the top Service right corner of the article or click here.

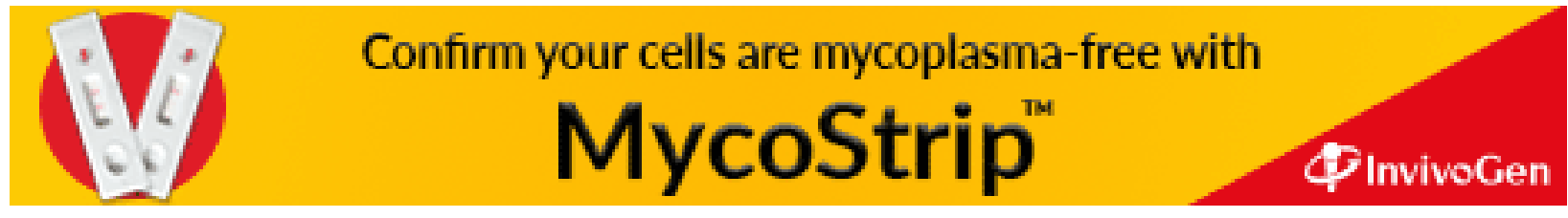

